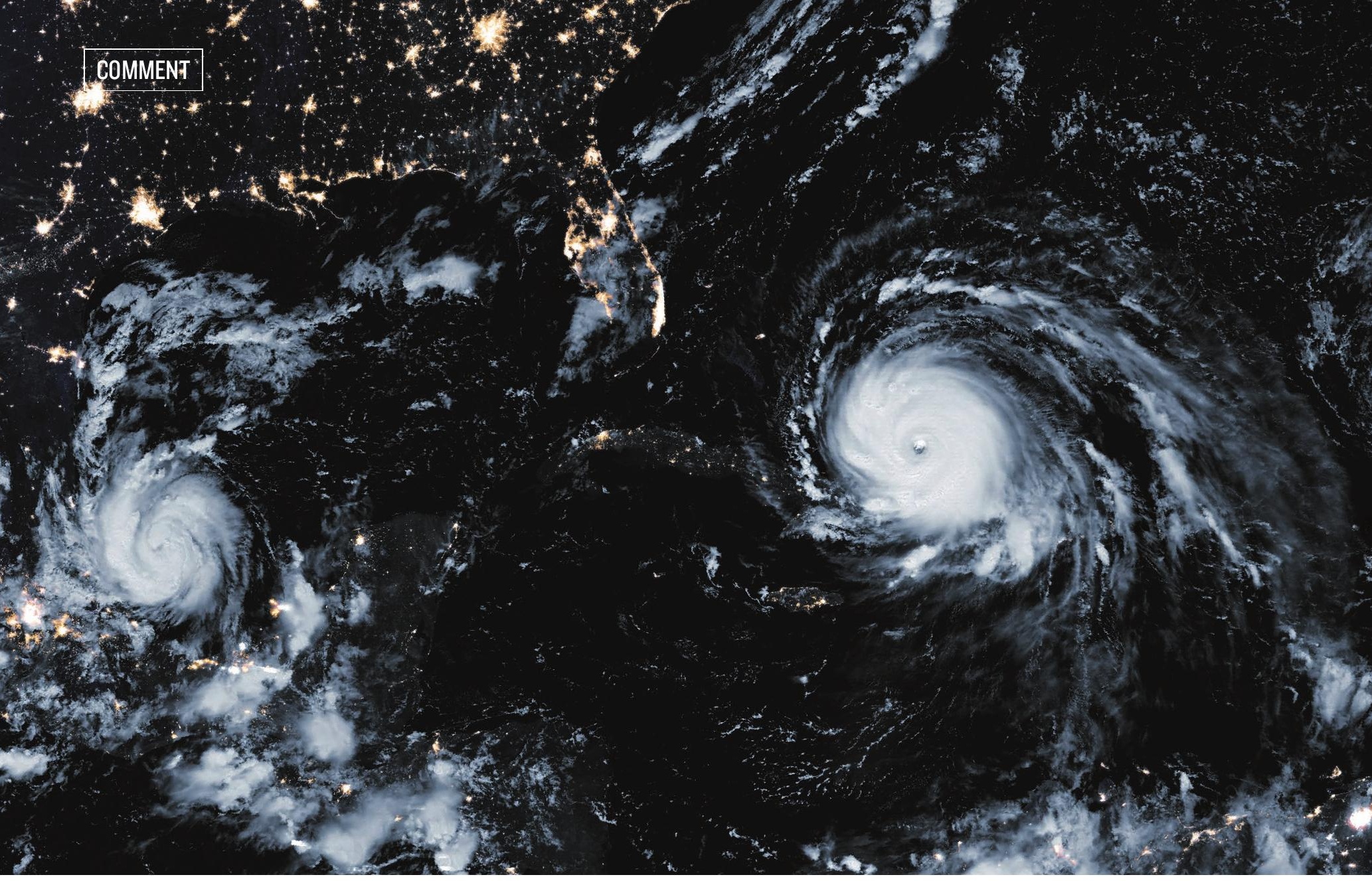

\title{
Five priorities for weather and climate research
}

\author{
Adapt to how data are made and used, urge Øystein Hov and colleagues.
}

$\mathrm{M}$ eteorology is entering a new era. Demand is growing worldwide for forecasts of storms, floods and droughts. For example, warnings of this year's devastating series of hurricanes (Harvey, Irma and Maria) across the western Atlantic issued by the World Meteorological Organization (WMO) Regional Specialized Centre in Miami, Florida, gave residents in affected areas time to seek shelter and take steps to lessen the impacts.

The risks and consequences of environmental change are rising. In 2016, insurance companies reported losses of US $\$ 175$ billion worldwide owing to natural catastrophes. Three-quarters of the losses were caused by meteorological, hydrological or climatological events.

Modes of collecting and delivering weather and climate information are evolving. Businesses and non-profit organizations are increasingly supplying weather services.
And data now stem from a broader range of sources, such as mobile-phone apps and smart devices.

The meteorological community must adapt. Two-thirds of national meteorological organizations need to strengthen their core infrastructure. Researchers need to adopt fresh approaches to collecting and disseminating environmental information. Communication, alliances and partnerships must be revamped to be interactive, informal and inclusive.

To begin this process, the WMO convened more than 100 experts in atmospheric, hydrological and related sciences in Geneva, Switzerland, in October. In a timely reminder of the threat of natural hazards, the meeting had to be relocated from Bali, Indonesia, because the Mount Agung volcano became active. The outcomes of the meeting have now been agreed by more than 50 countries and will be put to the World Meteorological
Congress for ratification in 2019.

As the organizing committee of the WMO science summit, here we highlight the five priorities that were agreed.

\section{DELIVER SCIENCE FOR SERVICES}

Weather forecasts, warnings and environmental information are increasingly provided by companies, non-governmental organizations and other bodies. For example, a public-private partnership in Indonesia runs the SMARTseeds phone app, which delivers weather forecasts and information on water availability to local growers of chillies, tomatoes and cucumbers.

Information must be tailored to reach the right person in the right form at the right time. For example, hydrologists need historical climate data and hourly predictions of rainfall to build models of urban flood risks. City managers want alerts of heavy rain weeks to days in advance. Once it hits, they need 


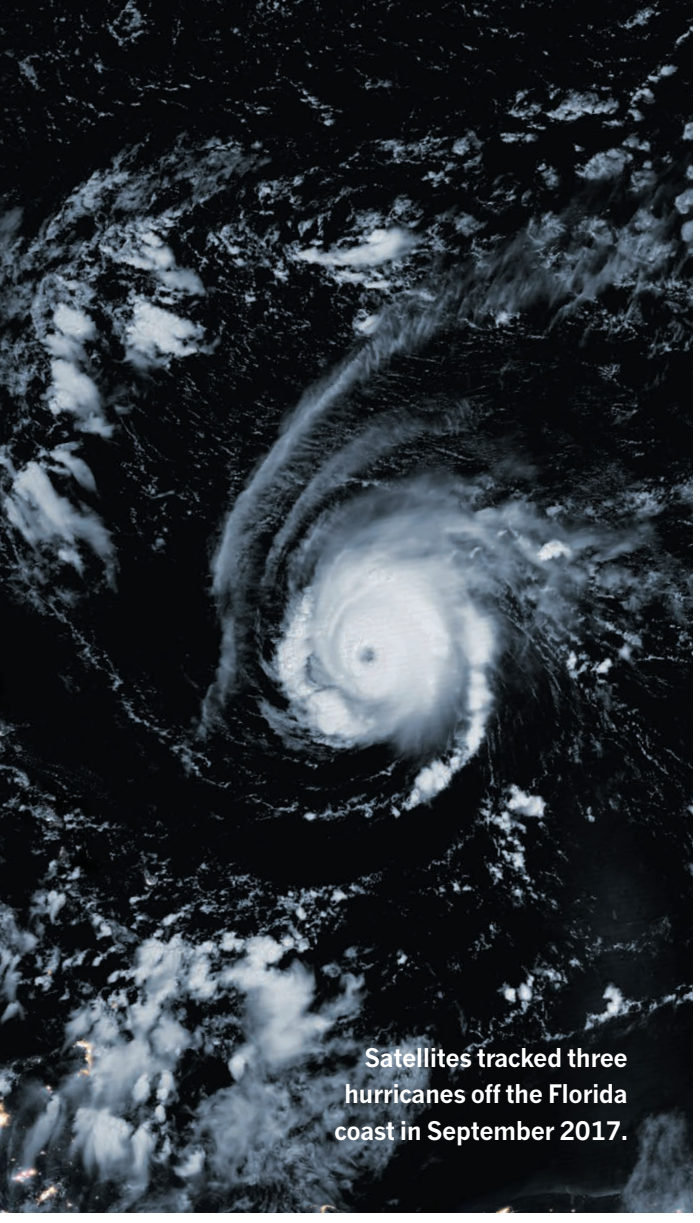

real-time monitoring of weather, traffic and vulnerable infrastructure. Official responders want to know under what circumstances it is more effective to evacuate people, divert waters or open shelters. The US National Weather Service, for example, develops tools to support decision-makers, including models of storm-surge inundation along coasts when a hurricane makes landfall.

It is hard to translate science into services. Researchers need to make their results more accurate, useful and readily available. Data are published in specialist terms and may not be relevant or robust enough to support reliable services. Knowing the details of how sea ice interacts with seawater or sunlight does not necessarily tell a ship owner how to operate fleets in the Arctic Ocean, for instance. Disciplines function in isolation, and need to work more closely with operational centres. Even the protocols used to share data differ between the weather and climate communities.

Meteorologists need to agree on the most relevant variables for different circumstances. For instance, local winds might be more important than atmospheric pressures for predicting urban air quality. Regional contexts must also be considered - weather information in east Asia, for instance, might need to include the complex hydrological cycle of the Tibetan Plateau.

It can be unclear who the key players are. Building a storm- or hurricane-warning system might involve researchers, system developers, communication experts, local government officials and community members. Disparate groups need mechanisms for articulating their requirements.

Private, public and academic institutions, together with non-governmental organizations, need to agree a strategy for translating and transmitting information. The WMO should create an environment that fosters joint research activities. Sources of funding must be identified for the research this strategy will require, as well as a process for prioritizing applications. Improving urban services is a pressing area, for example.

\section{BUILD SEAMLESS MODELS}

Predictive models currently consider the atmosphere, ocean, land and cryosphere separately. A combined model would improve the accuracy of forecasts by tapping connections between planetary processes on many scales, locally to globally and over minutes to centuries. The influences of tropical processes on the weather at mid-latitudes or the effects of city blocks on urban wind flows should be incorporated into products. Crowd-sourced data should be used.

A scientific strategy will be needed for merging models and data. It will have to work across all stages of information provision: from monitoring and observation to data assimilation, models, forecasting, dissemination and communication, perception and interpretation, decision-making and the creation of products ${ }^{1}$. Again, priority topics, such as the water cycle and urban development, could provide a starting point.

Efforts are beginning to combine models across domains - from weather to climate and from hydrology to air quality - through collaborations among the WMO's World Weather Research Programme ${ }^{2}$, World Climate Research Programme and Global Atmosphere Watch Programme ${ }^{3}$, and others. More groups need to be brought in, including hydrologists, social scientists, re-insurance companies and private foundations.

\section{IMPROVE INFRASTRUCTURE}

Information providers, modellers and users need more advanced computing infrastructures and facilities. These should be able to analyse exabytes $\left(10^{18}\right.$ bytes $)$ of data in real time and turn them into services ${ }^{4}$. Models must be upgraded to exploit multi-processor, cloud-based and distributed computing.

Access to data, tools and infrastructure needs to be broadened, especially in developing countries. For example, data and information from the European Commission's Copernicus projects, which are tailored for climate and air-quality services, need to be made more accessible. Fair use of data should be encouraged, such as by citing the originators of the data, methods and tools.

Observing networks must be extended to capture more relevant phenomena. Water fluxes at the interface between ocean, ice and atmosphere, or between land surface and groundwater, need to be better tracked. A methodology is needed to combine data of different qualities and types.

Technological shifts in environmental sensing, such as mapping pollution with mobile sensors, should be embraced. Crowdsourced observations must be included, such as data on the speeds of windscreen wipers (indicating rain intensity), which some car companies collect. Standards and protocols should be advanced through the WMO Integrated Global Observing System, together with interoperable platforms.

New models of public-private cooperation will be needed. For example, the WMO works with aviation companies to gather more than 700,000 observations each day of air temperature, wind speed and direction. Increasingly, it is also collecting measurements of humidity and turbulence in this way.

Long-term data storage is needed for measurements and models. Virtual and physical archives must be set up and shared, such as the Subseasonal-to-Seasonal (S2S) Prediction Project database that is hosted by the European Centre for Medium-Range

"Improving
urban services
is a pressing
area."

Weather Forecast and the China Meteorological Administration. Archives must be maintained for major initiatives such as the 2017-19 Year of Polar Prediction and 2017-19 Years of the Maritime Continent, which are improving predictions in polar and tropical regions, respectively.

\section{NURTURE A DIVERSE WORKFORCE}

Today's students and early-career scientists are the future of the field. Their education and training must become more interdisciplinary, service-focused and global in outlook $^{5}$. National meteorological and hydrological services and universities should collaborate to help early-career scientists to work on data and tools. The WMO's training activities should be updated and made available to a broader range of participants, for example through online tools. The private sector and users of data need to be integrated into a new model of training and mentoring.

Female scientists and those in developing countries need support. According to the United Nations Educational, Scientific and Cultural Organization (UNESCO) Institute for Statistics, the global average proportion of female researchers across all scientific fields is around $29 \%$. In many countries, around half of early-career 
meteorologists are women, but numbers decline along the career path. The roadblocks to gender equality need to be identified, and more meteorological service organizations should put strategies in place to retain female staff. Measures might include active recruiting and promotion of women, increasing flexibility and mobility, and stamping out bias.

The WMO also needs to know more about the factors that drive the decisions of earlycareer scientists who have different cultural backgrounds. What challenges do they face and what would help them to thrive?

The WMO needs to work more closely with existing networks. For example, the Earth Science Women's Network has more than 2,000 members in 50 countries and the Young Earth System Scientists community comprises more than 1,000 members in 80 or so countries.

The WMO should facilitate the use of scientific data and tools, including access to cloud-computing facilities, especially in developing countries. Regional centres of excellence need to be set up. For example, the China Meteorological Administration offers training through the Nanjing University of Information Science and Technology, and has established a joint office with the WMO to collaborate on high-impact weather events.

\section{SHARE IDEAS}

New networks will have to be set up to allow people with different kinds of knowledge and ways of tackling problems to share ideas. Smooth interactions should be encouraged by involving stakeholders in short-term projects of direct relevance to them. They could then feed back in to longer-term planning. For example, satellite developers, researchers and users of space data have cooperated to improve the use of observations, such as those for convective clouds, to better understand, model and predict the Earth system. Satellite data, which originally could be used only in clear-sky conditions, can now be included in models for cloudy and rainy areas, significantly increasing the capacity to simulate cloud properties.

Better links should be established between the WMO and related initiatives, such as regional projects fostered by international scientific platforms (including the International Council for Science, Future Earth and the UNESCO Intergovernmental Oceanographic Commission) and international donors (such as the Green Climate Fund run by the UN Framework Convention on Climate Change). The opportunities of working with the private sector need to be examined. These include improving monitoring and observation, models, forecasting, dissemination and communication, perception and interpretation, decision-making

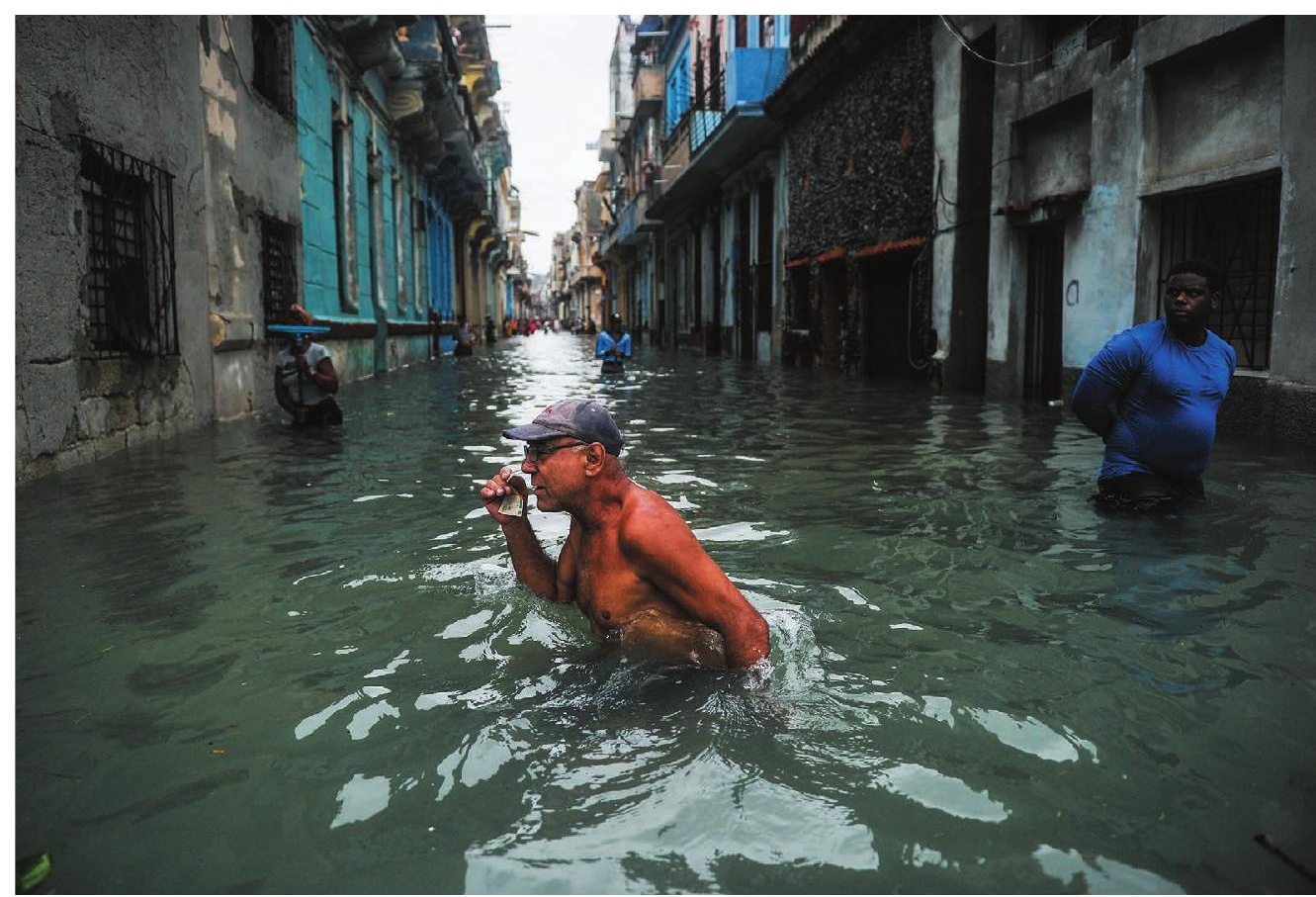

The streets of Havana, Cuba, were flooded after Hurricane Irma hit in September.

and end-user products. The risks, such as limiting the exchange of observations, must also be considered.

One of the WMO's strengths is its ability to develop long-term projects on fundamental research questions. For example, the S2S Prediction Project has improved seasonal predictions on timescales from weeks to months, which has enabled earlier management decisions to be made in areas including agriculture and food security, waterdisaster risk reduction and health ${ }^{6}$. More short-term projects, such as the 12 months of

\section{“Today's students and early-career scientists are the future of the field."}

targeted observations during the Year of Polar Prediction, would accelerate technological development and networking. Mechanisms are needed for prioritizing projects and developing long-term strategic plans with wide consultation.

The UN Sustainable Development Goals, the Paris climate agreement and the Sendai Framework for Disaster Risk Reduction should be used as catalysts to assemble the best talent, explore business and technology areas, and build collaborations.

Regional needs should be included through collaborations between national meteorological services and academic institutions. For example, the HIGHWAY project run by the WMO and supported by the UK Department for International Development is improving early warnings in the Lake Victoria basin in Africa by linking meteorological services and universities in five surrounding countries.

The weather, climate and environment communities should support the WMO's effort to meet the growing needs of society. .

Øystein Hov is president of the Commission for Atmospheric Sciences at the World Meteorological Organization (WMO), Geneva, Switzerland; and secretary general of the board of the Norwegian Academy of Science and Letters, Oslo, Norway. Deon Terblanche is director of research at the WMO. Gregory Carmichael is professor of chemical and biochemical engineering at the University of Iowa, Iowa City, USA. Sarah Jones is head of research and development and an executive board member at the German Meteorological Service (DWD), Offenbach, Germany. Paolo M. Ruti is chief of the World Weather Research Division at the WMO. Oksana Tarasova is chief of the Global Atmosphere Watch Division at the WMO.

e-mail:pruti@wmo.int

1. World Meteorological Organization. Seamless Prediction of the Earth System: from Minutes to Months (eds Brunet, G., Jones, S. \& Ruti, P. M.) (WMO, 2015); available at http://go.nature. com/2j59dxk

2. WMO. Catalysing Innovation in Weather Science: the World Weather Research Programme Implementation Plan, 2016-2023 (WMO, 2017); available at http://go.nature.com/2aj9b8o

3. WMO. WMO Global Atmosphere Watch Implementation Plan, 2016-2023 (WMO, 2017); available at http://go.nature.com/2bcwfc2

4. Bauer, P., Thorpe, A. \& Brunet, G. Nature $\mathbf{5 2 5}$ 47-55 (2015).

5. Rauser, F. et al. Bull. Am. Meteorol. Soc. http:// dx.doi.org/10.1175/BAMS-D-16-0025.1 (2017)

6. Vitart, F. et al. Bull. Am. Meteorol. Soc. http:// dx.doi.org/10.1175/BAMS-D-16-0017.1 (2017). 